\title{
The effect of distractions in the operating room during endourological procedures
}

\author{
Marjolein C. Persoon • Hans J. H. P. Broos • \\ J. Alfred Witjes • Ad. J. M. Hendrikx • \\ Albert J. J. M. Scherpbier
}

Received: 27 January 2010/ Accepted: 8 June 2010/Published online: 24 August 2010

(c) The Author(s) 2010. This article is published with open access at Springerlink.com

\begin{abstract}
Background Professionals working in the operating room (OR) are subject to various distractions that can be detrimental to their task performance and the quality of their work. This study aimed to quantify the frequency, nature, and effect on performance of (potentially) distracting events occurring during endourological procedures and additionally explored urologists' and residents' perspectives on experienced ill effects due to distracting factors.

Methods First, observational data were collected prospectively during endourological procedures in one OR of a teaching hospital. A seven-point ordinal scale was used to measure the level of observed interference with the main task of the surgical team. Second, semistructured interviews were conducted with eight urologists and seven urology residents in two hospitals to obtain their perspectives on the impact of distracting factors.

Results Seventy-eight procedures were observed. A median of 20 distracting events occurred per procedure, which corresponds to an overall rate of one distracting event every $1.8 \mathrm{~min}$. Equipment problems and procedurerelated and medically irrelevant communication were the most frequently observed causes of interruptions and
\end{abstract}

M. C. Persoon ( $\varangle)$ · H. J. H. P. Broos - Ad. J. M. Hendrikx Department of Urology, Catharina Hospital Eindhoven, Eindhoven, The Netherlands

e-mail: mleinpersoon@hotmail.com

M. C. Persoon - H. J. H. P. Broos - Ad. J. M. Hendrikx ·

A. J. J. M. Scherpbier

Department of Educational Development and Research,

Maastricht University, Maastricht, The Netherlands

J. A. Witjes

Department of Urology, University Medical Centre,

Nijmegen, The Netherlands identified as the most distracting factors in the interviews. Occurrence of distracting factors in difficult situations requiring high levels of concentration was perceived by all interviewees as disturbing and negatively impacting performance. The majority of interviewees (13/15) thought distracting factors impacted more strongly on residents' compared to urologists' performance due to their different levels of experience.

Conclusion Distracting events occur frequently in the OR. Equipment problems and communication, the latter both procedure-related and medically irrelevant, have the largest impact on the sterile team and regularly interrupt procedures. Distracting stimuli can influence performance negatively and should therefore be minimized. Further research is required to determine the direct effect of distraction on patient safety.

Keywords Distraction - Interruption · Endourology · Training

Multiple other problems, besides procedural knowledge and psychomotor abilities, influence the performance of urological surgeons and residents in the operating room (OR) $[1,2]$. These factors include degree of motivation, lack of sleep, variability in patient pathology, and distractions in the work environment. Research has revealed negative effects of distractions on performance outcomes in other disciplines [3-5].

Several studies have shown that distractions and interruptions are common in the OR. Most distractions are caseirrelevant conversations, telephone calls, pagers, music, and people entering the OR [6-8]. The effects of distracting stimuli on patient outcome variables are difficult to measure since complications occur infrequently and 
randomized studies in patients are unethical. However, distracting events have been shown to impact performance of technical skills in experimental settings. Hsu et al. [9] showed that distraction had a negative effect on performance of a peg transfer task on a box trainer in basic laparoscopic skills training and that this effect was stronger for novices than for experts. In addition, Pluyter et al. [10] demonstrated a decline in task score and an increase in task errors and operating time when a laparoscopic task was performed under distracting conditions.

Real-time studies of the impact of distractions in surgery are scarce. Previous studies described only small numbers within a large variety of procedures [6, 7]. Because of the proven negative effect of distracting events in other disciplines and in experimental settings, it is of paramount importance to explore the occurrence and impact of distraction in real-time surgical procedures. Given the limitations of quantitative methods for this type of study, we conducted a qualitative study using observations and interviews [11].

The aim of this study was to quantify the frequency, nature, and effects of distractions on interruptions of endourological procedures: How often does distraction occur in the OR? What is the nature of the distractions and how do they affect the task performance of the sterile team? Furthermore, urologists' and residents' perspectives on the experienced hindrance, possible effects of distracting factors, the need for restrictions, and their opinion on preparation in a skills lab were explored with semistructured interviews.

\section{Materials and methods}

We observed urology specialists and residents during performance of common endourological procedures [transurethral resection of the prostate (TURP), transurethral resection of a bladder tumor (TURBT), and ureteroscopic stone treatments (URS)] in a teaching hospital in the Netherlands. Conventional and laparoscopic procedures were excluded because they are different kinds of procedures requiring different instruments and techniques. Permission for the study was obtained from the institution's ethics committee. A distracting stimulus was defined as any event that can cause diversion from the task at hand, and a distraction was as any observed behavior indicating orientation away from the main task. An interruption was defined as when a distraction leads to a break in main task activity [6].

\section{Observations}

Data were collected between January and July 2009. During the first part of the study four urologists and three
Table 1 Observed distracting stimuli

\begin{tabular}{|c|c|}
\hline Source & Definitions \\
\hline Pager & Any pager activated in the operating room \\
\hline Telephone & $\begin{array}{l}\text { Any phone call made or received in the } \\
\text { operating room }\end{array}$ \\
\hline Radio & Action or response related to the radio \\
\hline Door movement & $\begin{array}{l}\text { One door movement: opening and closure of } \\
\text { a door in the operating room }\end{array}$ \\
\hline Equipment & Any item of equipment not at hand or falling \\
\hline Procedure & $\begin{array}{l}\text { Any conversation between team members } \\
\text { relevant to the procedure }\end{array}$ \\
\hline $\begin{array}{r}\text { Patient-irrelevant } \\
\text { communication }\end{array}$ & $\begin{array}{l}\text { Any conversation irrelevant to the case, but } \\
\text { relevant to other medical tasks in the } \\
\text { hospital }\end{array}$ \\
\hline $\begin{array}{l}\text { Medically irrelevant } \\
\text { communication }\end{array}$ & Any conversation with no medical relevance \\
\hline
\end{tabular}

residents were observed during endourological procedures in the same OR. Positioning of equipment and location of the sterile field were comparable in all cases. Data collection started when the urologist or resident put on sterile gloves and ended when the gloves were removed. Table 1 shows the different types of distracting stimuli. A sevenpoint ordinal scoring list, previously described by Healey et al. [6], was used to categorize distracting stimuli and interruptions that affect sterile team members (Table 2). Higher scores indicate a stronger impact on the flow of the

Table 2 Seven-point ordinal scale and the related effect of the distracting stimulus

\begin{tabular}{|c|c|}
\hline Rating & Observed effects \\
\hline 1 & $\begin{array}{l}\text { Potentially distracting stimuli: events with the potential to } \\
\text { distract the sterile team }\end{array}$ \\
\hline 2 & $\begin{array}{l}\text { Sterile team member momentarily distracted: possible } \\
\text { involvement of a single sterile member in an event not } \\
\text { related to the primary task, e.g., a short head turn in } \\
\text { response to a visual or auditory stimulus }\end{array}$ \\
\hline 3 & $\begin{array}{l}\text { Sterile team member engages in distraction: similar } \\
\text { distraction in } 2 \text {, but the sterile member engages with the } \\
\text { source of distraction by verbally responding while } \\
\text { maintaining primary task activity (multitasking) }\end{array}$ \\
\hline 4 & $\begin{array}{l}\text { Sterile team member's primary task interrupted: a single team } \\
\text { member ceases his/her current tasks to engage entirely in } \\
\text { the distracting stimulus }\end{array}$ \\
\hline 5 & $\begin{array}{l}\text { Sterile team momentarily distracted: two or more sterile team } \\
\text { members respond to a stimulus with a short head turn, no } \\
\text { verbal response }\end{array}$ \\
\hline 6 & $\begin{array}{l}\text { Sterile team engage in secondary tasks: two or more team } \\
\text { members engage with the source of distraction by verbally } \\
\text { responding while maintaining primary task activity }\end{array}$ \\
\hline 7 & $\begin{array}{l}\text { Sterile team's work interrupted-operation flow disrupted: } \\
\text { interruption of the current primary task of the sterile team, } \\
\text { the operation flow is disrupted }\end{array}$ \\
\hline
\end{tabular}


procedure. A score of 1 represents a potentially distracting stimulus not followed by a response. The highest score, 7, represents a stimulus that causes complete interruption of the primary task of the sterile team and disruption of the flow of the procedure. For each potentially distracting stimulus, frequency and severity of effect were measured. In addition, which member of the surgical team that caused the distracting event it was recorded, with a distinction made between nonsterile and sterile team members, i.e., urologists, residents, and sterile nurses.

Prior to the actual study, we conducted a pilot study in which six endourological procedures (not included in the final study) were observed and rated in order to familiarize the researchers with the scoring list and to standardize practical issues such as the positioning of the observer in the OR for optimal registration of potentially distracting stimuli. The first 17 procedures of the actual study were observed by two independent observers: a researcher and an intern. Interobserver agreement, calculated as the number of agreements divided by the sum of agreements and disagreements multiplied by $100 \%$, was $80 \%$ during the first 17 procedures [12]. Thereafter, the procedures were observed by the intern who was in his surgical rotation in the last year of the 6-year curriculum. Staff members were used to seeing the intern in the OR as an observer. The observer did not participate in the procedure to minimize the impact on the environment under study [11]. The OR team was informed that the intern was doing a research project investigating the logistics and the division of tasks during surgical procedures.

\section{Semistructured interviews}

After completing all the observations, the researcher and the intern conducted semistructured interviews with urology specialists and residents in two teaching hospitals, exploring their thoughts on how distracting factors affect task performance of the surgical team. They were asked about personal experiences with different kinds of distraction and the usefulness of preparation in a skills lab. The interview was guided by 13 questions and by any new questions prompted by issues brought up during interviews. After explaining the aim of the study, informed consent was obtained. Semistructured interviews were used because they enable interviewees to talk about the effects of distraction in detail and depth and at the same time ensure that a predefined list of topics is addressed during each interview [13]. All conversations were recorded on tape. The two interviewers analyzed the interviews independently and discussed their analysis to ensure similarity of interpretation. The responses were categorized by five themes: sources of distraction, situations in which distraction is the most annoying, impact of distraction on level of performance, the need for measures to limit distractions in the OR, and the usefulness of preparation in a skills lab.

\section{Results}

A total of 78 endourological procedures were observed: 27 TURBTs, 26 TURPs, and 25 URSs. Time to completion ranged from 8 to $107 \mathrm{~min}($ median $=35 \mathrm{~min})$. No serious intraoperative complications occurred.

\section{Frequencies}

The total number of distracting events observed per procedure ranged from 2 to 53 (median $=20$ ). Completion time of the procedures varied and the overall mean rate of occurrence of distracting stimuli was 1 every $1.8 \mathrm{~min}$. Table 3 shows the mean frequencies and the team member involved for each source of distraction per procedure. Of all observed sources of distraction, an activated pager had the lowest (0.41) and door openings had the highest

Table 3 Frequency of each source of distraction induced by each team member separately

\begin{tabular}{|c|c|c|c|c|c|}
\hline & \multirow{2}{*}{$\begin{array}{l}\text { Number of occurrences } \\
\text { per procedure }\end{array}$} & \multicolumn{4}{|c|}{ Mean $\%$ of occurrences induced by } \\
\hline & & SU & SR & $\mathrm{SN}$ & NST \\
\hline Pager & 0.41 & 44 & 25 & 0 & 31 \\
\hline Telephone & 1.50 & 8 & 0 & 1 & 91 \\
\hline Radio & 2.62 & 31 & 22 & 24 & 23 \\
\hline Door movement & 8.85 & 0 & 1 & 0 & 99 \\
\hline Procedure-related communication & 1.65 & 19 & 19 & 19 & 43 \\
\hline Patient-irrelevant communication & 1.26 & 16 & 4 & 28 & 52 \\
\hline Medically irrelevant communication & 2.13 & 16 & 1 & 30 & 53 \\
\hline Equipment & 1.72 & 0 & 0 & 0 & 0 \\
\hline
\end{tabular}

$S U$ sterile urologist, $S R$ sterile resident, $S N$ sterile nurse, $N S T$ nonsterile team member 
Table 4 Mean rating of each source of distraction induced by each team member, calculated

$S U$ sterile urologist, $S R$ sterile resident, $S N$ sterile nurse, $N S T$ nonsterile team member from all 78 procedures

\begin{tabular}{llllll}
\hline & SU & SR & SN & NST & Rating per event \\
\hline Pager & 3.67 & 4.1 & 0 & 1.50 & 2.31 \\
Telephone & 2.50 & 0 & 1.00 & 2.07 & 1.39 \\
Radio & 1.38 & 1.06 & 1.11 & 1.02 & 1.14 \\
Door movement & 0 & 1.00 & 0 & 1.56 & 1.28 \\
Procedure-related communication & 5.68 & 5.54 & 4.69 & 3.27 & 4.80 \\
Patient-irrelevant communication & 4.95 & 4.17 & 4.02 & 3.67 & 4.20 \\
Medically irrelevant communication & 5.55 & 6.00 & 4.34 & 3.28 & 4.79 \\
Equipment & - & - & - & - & 4.97 \\
\hline
\end{tabular}

process. On the other hand, distractions like good music can contribute to a good work environment, which has a positive impact on performance.

Situations in which distraction is the most annoying

All interviewees reported being the most annoyed when they were distracted during difficult and stressful situations requiring high levels of concentration. Urologists and residents believed they were better able to deal with distractions during routine procedures without problems. Consequently, $87 \%$ of the interviewees thought distraction impacted residents more strongly than experienced urologists, as one interviewee said:

Experienced urologists compared to residents are more in control when unexpected difficulties occur.

They are better able to influence the environment to optimize the working climate.

Four urologists and one resident said that individual characteristics of residents and urologists had an important effect on the degree to which distractions affected performance.

Impact of distraction on level of performance

Eight urologists, with at least 5 years of experience in unsupervised performance of endourological interventions, and seven residents in two teaching hospitals were interviewed. The residents were in their third to sixth year of the 6-year urology program.

\section{Sources of distraction}

Both urologists and residents said that distractions mostly involved equipment not being at hand or failing, caseirrelevant communication surrounding the sterile field, and having to respond to questions about another case. Music was considered to be distracting by only two interviewees and some found it helpful in relieving stress. One urologist said:

Emptiness of water supply and malfunctioning of the resectoscope often results in disruption of the surgical
All interviewees stated that distraction could have a negative effect on the quality of surgical performance. However, none of them thought interruptions during a procedure compromised patient outcomes although they could slow performance and result in secondary tasks. As one of the residents put it:

Distracting events that irritate me can affect my level of performance negatively. On the other hand, when I am aware of being distracted, I ask for silence in the OR. I don't think there is a negative effect for the patient.

Need for restrictions

The vast majority of the participants stated that distracting stimuli should be minimized in the OR. According to $87 \%$ 
of the participants, it is mainly the responsibility of the urologist or resident performing the procedure to create an optimal environment by asking for silence when they are being distracted. However, as one resident stated:

It depends on the relationship between resident and supervisor how free you feel to make comments on things like distraction during a procedure.

A protocol with measures to reduce disturbance, such as reducing door movements, removing pagers from the OR, and implementing a code of conduct, were mentioned as measures to create an optimal work environment.

\section{Usefulness of preparation in a skills lab}

On this point there was no consensus. None of the residents and only two of the urologists saw a benefit to training in distracting circumstances outside the OR to prepare for real performance in the OR. However, the majority of participants (73\%) thought training in technical skills in a skills lab was good preparation for real-time surgery. In this way, routine performance is attained faster, which results in diminished susceptibility to distracting events in the OR. Others were skeptical about skills lab training since not all circumstances can be simulated. One urologist said:

Specific training of skills could be indirectly useful as a preparation to perform in a distracting environment but it is not possible to prevent all distracting events.

\section{Discussion}

Distraction has a proven negative impact on performance and human safety outside medicine [3-5, 12] and also on surgical tasks in experimental settings $[9,10]$. In this study, the impact of distraction and interruption on endourological procedures was investigated by measuring the frequency and impact of distraction and interruptions in one $\mathrm{OR}$ and by exploring the perceptions of urologists and residents. Distraction occurred at a mean frequency of once every $1.8 \mathrm{~min}$. Equipment problems, procedure-related communication, patient-irrelevant communication, and medically irrelevant communication have the strongest impact on the flow of the operation. Door openings and pagers occurred very frequently but did not in the least distract the sterile team. This is consistent with the results of the interviews. Both urologists and residents believe that distractions are less disturbing if the difficulty of the endourological task lies within their range of technical skills compared to more challenging procedures. They also unanimously conclude from this that the effect of distracting stimuli is stronger in residents who are in their learning curve. This is supported by an in vitro study by Hsu et al. [9] in which the performance of experienced surgeons, in contrast to that of novices, was not affected by cognitive distraction. This may be because experienced surgeons have attained a level of automatic task performance that is impervious to distractions. This suggests that training technical skills to a high level of performance outside the OR could minimize the effect of distraction on performance during real-time surgery. However, in the interviews only two urologists thought preparation in a skills lab could be useful for diminishing susceptibility to distracting events in the OR.

OR personnel outside the sterile team are the most frequent sources of distraction due to communication, whether it is procedure-related, patient-irrelevant, or medically irrelevant. All but two of the interviewees said it was the responsibility of the urologist to deal with distracting events. This means that the main surgeon should ask for silence when this is appropriate. This can be more difficult for residents than for urologists since interpersonal relationships and hierarchical structure determine to what degree they can influence the environment. Awareness of the presence of distracting stimuli and their potentially negative effect on the flow of procedures is therefore an important factor in dealing with distraction. As a first step, a protocol of restrictive measures to prevent distractions seems inevitable.

Pluyter et al. [10] refer to the more complex environment in the OR during procedures involving multidimensional information technologies (IT) such as minimally invasive surgery. This may put extra physical and cognitive demands on the medical staff and cause errors. The hypothesis that dense IT usage is a source of distraction is supported by our study because we found that equipment problems were one of the main sources of distraction that could interrupt endourological procedures in which more equipment and technology are used compared to open surgery.

Not all potentially distracting stimuli seem to have a negative effect on surgical procedures [14, 15]. Ullmann et al. [8] evaluated the perceptions of physicians and nurses working in the OR with respect to the influence of music. Sixty-three percent of the responders believed that music had a positive effect on communications among staff, and $77 \%$ reported that music had a calming effect and made them more efficient. Moorthy et al. [16] concluded that surgeons can "block out" noise and music due to the high levels of concentration required for complex surgical tasks. Our results support this notion since only 2 of 15 participants thought music was distracting in certain situations. However, there is an important difference between a potentially distracting factor like background music and cognitive distraction caused by conversations. Findings in the field of cognitive psychology have shown that simultaneous performance of two or more tasks can slow 
execution times and increase errors compared to singletask performance. This notion is referred to as multitask interference and should be minimized [17, 18].

\section{Limitations of the study}

A limitation of the qualitative observation method used in this study is dependence on observers' ability to interpret events accurately and the possibility of variation between observers. Observers may identify and interpret events differently and may be biased. We therefore used two observers and made sure that acceptable interobserver agreement had been reached (80\%) before we allowed one observer to conduct observations on his own. Limitations of semistructured interviews should also be considered. Although we processed the data anonymously, it cannot be ruled out that interviewees were biased and gave socially desirable answers, especially when they had been observed and had been involved in distractions during observation [13]. In order to counter this effect, we also interviewed surgeons in another teaching hospital who had not participated in the observational part of the study. The absence of meaningful differences between the two groups of interviewees suggests that there was little or no bias due to observation or to differences in organizational structure between the two hospitals.

\section{Recommendations for further research}

Horberry et al. [19] determined that in-vehicle tasks impaired several aspects of driving performance and safety. In our study we observed no serious intraoperative complications that could be attributed to interruptions. However, our main focus was on distracting stimuli. Patient outcome is determined by complex interactions of many factors. It would therefore require larger numbers of procedures with standardized observation methods, e.g., video recordings, to further study the direct effects of distraction on patient safety.

We observed only endourological procedures in order to standardize the protocol. Further research should investigate distracting stimuli in laparoscopic and open surgery. More importantly, a protocol of measures inside and outside the OR should be implemented and examined to minimize the effect of distraction on the performance of surgeons.

\section{Conclusion}

Distracting events are a frequent occurrence in the endourological OR. Equipment problems and procedure-related communication and medically irrelevant communication have the strongest impact on the sterile team and regularly interrupt procedures. Distracting stimuli are conceived as influencing performance negatively and should be minimized in the OR. Further research is required to determine any direct effect of distraction on patient safety.

Acknowledgments The authors thank Mereke Gorsira for her editorial assistance.

Disclosures Drs. Persoon, Broos, Witjes, Hendrikx, and Scherpbier have no conflicts of interest or financial ties to disclose.

Open Access This article is distributed under the terms of the Creative Commons Attribution Noncommercial License which permits any noncommercial use, distribution, and reproduction in any medium, provided the original author(s) and source are credited.

\section{References}

1. Hall JC, Ellis C, Hamdorf J (2003) Surgeons and cognitive processes. Br J Surg 90:10-16

2. Kohls-Gatzoulis JA, Regehr G, Hutchison C (2004) Teaching cognitive skills improves learning in surgical skills courses: a blinded, prospective, randomized study. Can J Surg 47:277-283

3. Forster S, Lavie N (2008) Failures to ignore entirely irrelevant distractors: the role of load. J Exp Psychol Appl 14:73-83

4. McEvoy SP, Stevenson MR, McCartt AT, Woodward M, Haworth C, Palamara P (2005) Role of mobile phones in motor vehicle crashes resulting in hospital attendance: a case-crossover study. BMJ 331:428

5. McEvoy SP, Stevenson MR, Woodward M (2007) The contribution of passengers versus mobile phone use to motor vehicle crashes resulting in hospital attendance by the driver. Accid Anal Prev 39:1170-1176

6. Healey AN, Primus CP, Koutantji M (2007) Quantifying distraction and interruption in urological surgery. Qual Saf Health Care 16:135-139

7. Primus CP, Healey AN, Undre S (2007) Distraction in the urology operating theatre. BJU Int 99:493-494

8. Ullmann Y, Fodor L, Schwarzberg I, Carmi N, Ullmann A, Ramon Y (2008) The sounds of music in the operating room. Injury 39:592-597

9. Hsu KE, Man FY, Gizicki RA, Feldman LS, Fried GM (2008) Experienced surgeons can do more than one thing at a time: effect of distraction on performance of a simple laparoscopic and cognitive task by experienced and novice surgeons. Surg Endosc 22:196-201

10. Pluyter JR, Buzink SN, Rutkowski AF, Jakimowicz JJ (2010) Do absorption and realistic distraction influence performance of component task surgical procedure? Surg Endosc 24(4):902-907

11. Mays N, Pope C (1995) Qualitative research: observational methods in health care settings. BMJ 15:182-184

12. Mudford OC, Taylor SA, Martin NT (2009) Continuous recording and interobserver agreement algorithms reported in the Journal of Applied Behavior Analysis (1995-2005). J Appl Behav Anal 42: 165-169

13. Britten N (1995) Qualitative interviews in medical research. BMJ 22:251-253

14. Liu EH, Tan S (2000) Patients' perception of sound levels in the surgical suite. J Clin Anesth 12:298-302

15. Schneider AJ, Biebuyck JF (1990) Music in the operating-room. Lancet 9:1407 
16. Moorthy K, Munz Y, Undre S, Darzi A (2004) Objective evaluation of the effect of noise on the performance of a complex laparoscopic task. Surgery 136:25-30

17. Levy J, Pashler H (2001) Is dual-task slowing instruction dependent? J Exp Psychol Hum Percept Perform 27:862-869

18. Meyer DE, Kieras DE (1997) A computational theory of executive cognitive processes and multiple-task performance: part 1 . Basic mechanisms. Psychol Rev 104:3-65
19. Horberry T, Anderson J, Regan MA, Triggs TJ, Brown J (2006) Driver distraction: the effects of concurrent in-vehicle tasks, road environment complexity and age on driving performance. Accid Anal Prev 38:185-191 